\title{
Control of Drosophila adult pattern by extradenticle
}

\author{
Sergio González-Crespo and Ginés Morata
}

Centro de Biología Molecular, Consejo Superior de Investigaciones Científicas, Universidad Autónoma de Madrid, 28049 - Madrid, Spain

\section{SUMMARY}

The homeobox gene extradenticle (exd) acts as a cofactor of the homeotic genes in the specification of larval patterns during embryogenesis. To study its role in adult patterns, we have generated clones of mutant $\mathrm{xxd}^{-}$cells and examined their effect on the different body parts. In some regions, exd ${ }^{-}$clones exhibit homeotic transformations similar to those produced by known homeotic mutations such as Ultrabithorax (Ubx), labial (lab), spineless-aristapedia $\left(s s^{a}\right)$ or Antennapedia (Antp). In other regions, the lack of exd causes novel homeotic transformations producing ectopic eyes and legs. Moreover, exd is also required for functions normally not associated with homeosis, such as the maintenance of the dorsoventral pattern, the specification of subpatterns in adult appendages or the arrangement of bristles in the mesonotum and genitalia. Our findings indicate that $e x d$ is critically involved in adult morphogenesis, not only in the homeotic function but also in several other developmental processes.

Key words: cofactor, clonal analysis, homeotic genes, imaginal discs, human $p b x$ genes, Drosophila, extradenticle

\section{INTRODUCTION}

A small number of primary homeotic genes in the Antennapedia-Complex (ANT-C) and the Bithorax-Complex (BX-C) specify the identity of segments of Drosophila (Lewis, 1978; Sánchez-Herrero et al., 1985; Kaufman et al., 1990). Several lines of evidence suggest that there must be mechanisms to modulate the function of these primary homeotic genes in order to achieve the high degree of morphological diversity that we observe in the larva and the adult. For example, only the three genes of the BX-C (Ultrabithorax (Ubx), abdominal-A $(a b d-A)$ and Abdominal-B $(A b d-B))$ determine the identity of two thoracic and eight abdominal segments (Sánchez-Herrero et al., 1985). In addition, homeotic genes exhibit a developmental specificity that can be revealed by the concrete patterns that they determine when they act alone. For example, mutant larvae containing only $U b x$ function $\left(U b x^{+} a b d-A^{-} A b d-B^{-}\right)$have all abdominal segments with identity of the first abdominal one (A1), whereas $U b x^{-} a b d$ $A^{+} A b d-B^{-}$larvae, with only $a b d-A$ function, have abdominal segments of more posterior identity (Sánchez-Herrero et al., 1985; Casanova et al., 1987). Similarly, the ectopic expression of each of these homeotic genes in the absence of the rest of BX-C products results in gene-specific segment patterns (González-Reyes and Morata, 1990; SánchezHerrero et al., 1994).

The homeotic genes encode DNA-binding proteins with a homeodomain (Desplan et al., 1985; Hoey and Levine, 1988; Desplan et al., 1988) that can act as transcription factors (Thali et al., 1988) presumably regulating the transcription of sets of target genes, which are ultimately responsible for the developmental specificity. However, different homeotic proteins show similar DNA-binding specificities in vitro (Hoey and Levine, 1988) again posing the question as to how the morphological diversity is encoded.

One factor contributing to the specificity of homeotic gene function appears to be the product of the gene extradenticle (exd) (reviewed in Wilson and Desplan, 1995). Mutations in exd produce homeotic transformations in several larval segments without affecting the expression patterns of homeotic genes, suggesting that it acts as a cofactor of homeotic gene function (Peifer and Wieschaus, 1990). exd encodes a protein with a highly conserved homeodomain whose mRNA is broadly distributed in the embryo (Rauskolb et al, 1993). Recent molecular analyses have demonstrated that the exd and Ubx products can bind cooperatively to DNA and that the binding specificity of Ubx is modified by exd (Chan et al., 1994; van Dijk and Murre, 1994), thus providing further support to the cofactor hypothesis.

The exd gene has a maternal and a zygotic component that are equivalent since the paternal exd product can rescue the lack of the maternal one (Peifer and Wieschaus, 1990; Rauskolb et al, 1993). exd ${ }^{-}$larvae lacking maternal and zygotic products have most segments affected, but the full array of transformations is difficult to study because these larvae are grossly abnormal. In mutant larvae lacking only the zygotic component, discrete effects can be seen but these are only part of the phenotype (Peifer and Wieschaus, 1990).

The role of exd on the determination of adult patterns is not known. In adult flies, the homeotic domains are well known and defined in great detail (Sánchez-Herrero et al., 1985; Kaufman et al., 1990) as is the manner in which these patterns are sequentially generated during development (Cohen, 1993). Therefore, the role of exd in this process can be investigated. 
A particular advantage of studying the adult patterns is that the elimination of the zygotic component of exd during the larval period represents the complete loss of function of the gene because the maternal product perdures only to embryonic stage 9 (Rauskolb et al., 1993). Since null exd mutations are lethal, we have used mosaic analysis and the Minute technique (Morata and Ripoll, 1975) to generate large clones of exd ${ }^{-}$cells all over the body in $\operatorname{exd}^{+}$flies and built a piecemeal image of exd $d^{-}$adult patterns. We also show that the functional requirements for exd function correlate with the spatial distribution of the exd protein in imaginal discs.

\section{MATERIALS AND METHODS}

\section{Clonal analysis}

Mosaic analysis was performed using the null allele exd $\mathrm{XP11}$ (Rauskolb et al., 1993). Cell clones deficient for exd function were produced irradiating larvae of genotype $y$ exd $d^{X 11} f^{36 a} / M(1) o^{S p}$ at 24, 48, 96, 72 and 120 hours AEL with X-rays (1000 Rad). In a separate set of experiments, we irradiated $y w$ exd $^{X P 11} \beta^{36 a} / M(1) o^{S p}$ larvae under similar conditions. An event of mitotic recombination proximal to the locus forked $(f)$ resulted in a clone of cells homozygous for $\operatorname{exd}^{X P 11}$, yellow $(y)$ and $f^{36 a}$ in the first set of experiments and $e x d^{X P 11}, y$, white $(w)$ and $f^{36 a}$ in the second one. The mutations $y$ and $f^{36 a}$ mark cuticle structures (yellow cuticle and bristles and forked bristles, respectively) and $w$ marks the eye ommatidia (white eye) so that clone cells are easily distinguished from wild-type cells. The mutant clones also lose the retarding Minute condition and proliferate faster than surrounding wild-type cells (Morata and Ripoll, 1975). Depending on the experiment, the clones were initiated during the first, second or third larval period, but reach a large size even if induced relatively late in development so that the kind of pattern they produce can be easily diagnosed.

\section{Preparation of adult cuticle for microscopic examination}

Adult flies of the apropriate genotype were dissected in alcohol and cut in pieces, separating heads and dorsal and ventral thoracic and abdominal parts. These were subsequently treated with $10 \% \mathrm{KOH}$ to digest the internal tissues, washed with propanol and mounted in Euparal.

\section{Production of anti-exd antiserum}

Bacterial exd protein was prepared as a fusion with GST using the pGEX KG expression plasmid and the Escherichia coli strain HB101 as described (Smith and Johnson, 1988; Guan and Dixon, 1991). The GST-exd fusion protein contains the C-terminal 153amino-acid residues of exd, which spans the homeodomain and the C-terminal region. A polyclonal antiserum against this GST-exd fusion protein was raised in rats and used in immunolocalization experiments.

\section{Immunolocalization}

Imaginal discs from third instar larvae were dissected in PBS, fixed in $4 \%$ paraformaldehyde in PBS during 20 minutes and in 4\% paraformaldehyde, $0.1 \%$ Triton $\mathrm{X}-100$ and $0.1 \%$ sodium deoxycholate in PBS during 20 minutes, briefly washed in PBS and blocked in $1 \%$ BSA, $0.3 \%$ Triton X-100 during 80 minutes. Discs were then incubated in preabsorbed anti-exd antiserum diluted $1 / 400$ to $1 / 1000$ overnight at $4^{\circ} \mathrm{C}$. After washing and second blocking, discs were incubated in 1/400 biotinylated anti-rat $\operatorname{IgG}$ (Amersham) during 2 hours and processed for DAB staining using the Vectastain Elite ABC Kit (Vector). Stained discs were mounted in $80 \%$ glycerol and photographed under Normarski optics.

\section{RESULTS}

\section{exd function is autonomously required in imaginal cells}

We have generated $y$ exd ${ }^{-} f^{36 a} M^{+}$clones in different body parts and studied the requirements for exd function in imaginal cells during the different stages of the larval period. The mutant clones exhibit a mutant phenotype even if induced at the end of the third larval period. Clones from this stage are very small, made of few cells, indicating a requirement for exd function until the end of the larval period, just like the regular homeotic genes (Morata and Lawrence, 1977). Furthermore, as far as we can ascertain, all the mutant cells show mutant phenotype, even though they are surrounded by wild-type cells. This behavior indicates that exd function is cell autonomously required, another feature in common with regular homeotic genes. The examination of their phenotypic effects reveals that exd is necessary for a number of distinct adult patterns.

\section{In some regions, exd- clones exhibit a phenotype similar or identical to those produced by mutations in known homeotic genes}

This behavior is shown by clones found in part of the metathoracic segment (T3) and of the head. A total of $27 \mathrm{exd}^{-}$clones were found in the metanotum (dorsoproximal region of T3) and they produce a transformation into mesonotum (dorsoproximal region of T2) (Fig. 1A), just like that produced by $U b x^{-}$clones in this region (Kerridge and Morata, 1982). In contrast, we did not find any clone showing transformation in the haltere appendage, even though both metanotum and haltere differentiate from cells of the haltere imaginal disc. The lack of detection of $\mathrm{exd}^{-}$clones in the haltere is most probably not due to cell lethality, but to the difficulty of scoring if they remain untransformed. These results indicate that $U b x$ function is mediated by that of exd in the metanotum, but not in the appendage portion of the segment. This is in contrast with the requirements for $U b x$, which extend to the entire segment (Kerridge and Morata, 1982). These results are consistent with observations demonstrating a molecular interaction between Ubx and exd products (Chan et al 1994; van Dijk and Murre, 1994), but suggest that the interaction is restricted to part of the $U b x$ domain in T3.

In the antenna, we detected a total of 30 clones and all show a transformation into distal leg closely resembling those produced by spineless-aristapedia $\left(s s^{a}\right)$ (Fig. 1B) or Antennapedia (Antp) (Fig. 1C) mutations (Postlethwait and Girton, 1974; Schneuwly et al., 1987). Finally, in the dorsal posterior head, exd ${ }^{-}$clones $(n=48)$ differentiate thoracic tissue (Fig. 1D) resembling the transformation reported for the loss of the labial gene (Merrill et al., 1989). Some of these clones (marked $y w f^{36 a}$ ) extend to the normal eye, where they differentiate normally. We have found that the great majority of these clones appear in the posterior head capsule, from the ocellar region toward the occipital region. Anterior to the ocellus, in the prefrons, for example, exd $d^{-}$clones are nearly always absent. Mutant clones may either behave as cell lethal in those regions or sort out from their site of origin and tend to occupy certain head regions.

\section{In some regions, exd- clones produce novel homeotic transformations}

In the A1-A4 abdominal tergites, all the 67 mutant clones examined present a pattern similar, though not identical, to that 

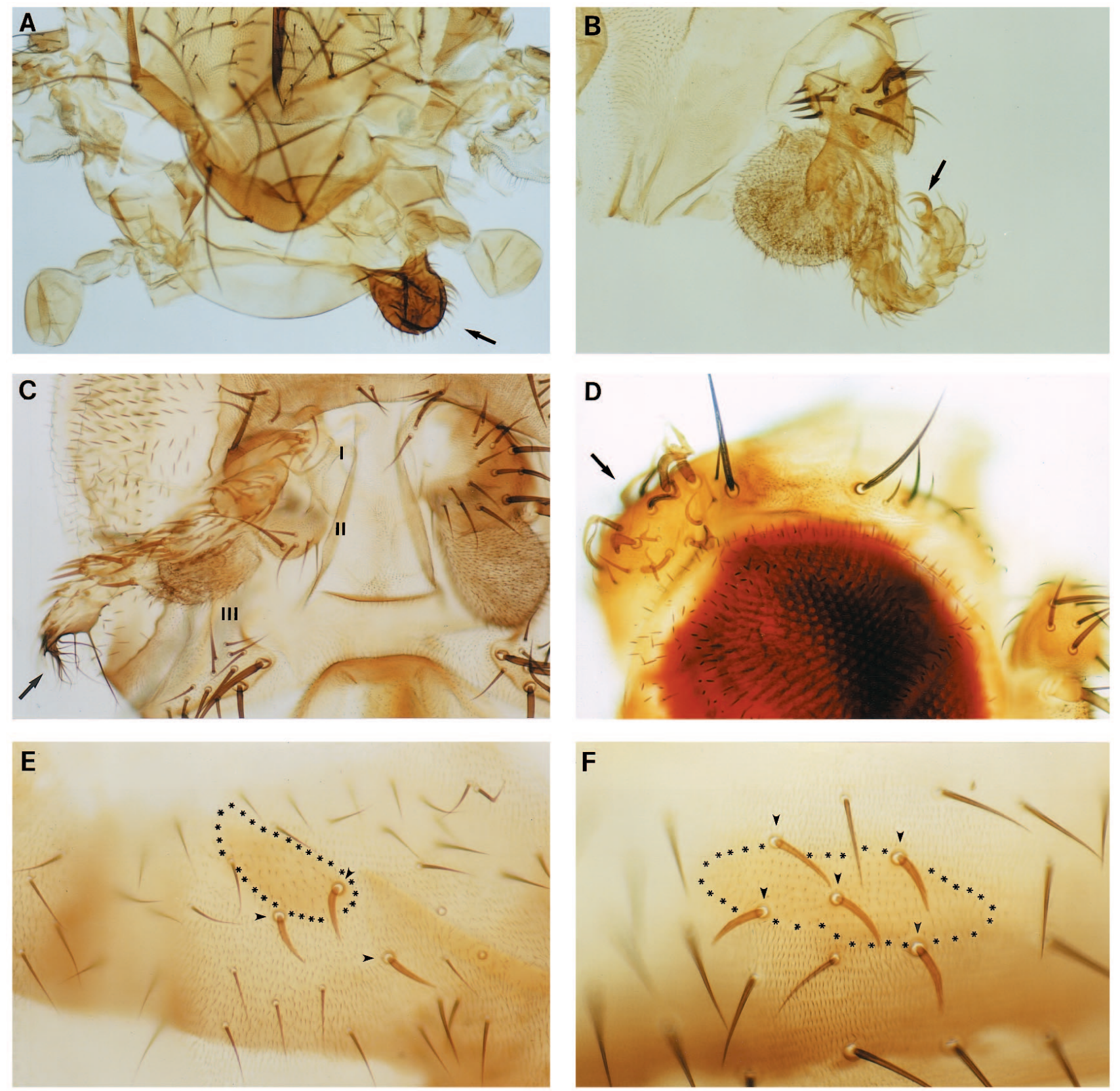

Fig. 1. Homeotic transformations produced by $e x d^{-}$clones. (A) $U b x$-like. Clone (arrow) transforming metanotum into mesonotum, just as $U b x^{-}$ clones do in this region (Kerridge and Morata, 1982). (B) $s s^{a}$-like. Transformation of the third antennal segment and the arista into distal leg structures, mimicking the $s s^{a}$ phenotype (Postlethwait and Girton, 1974). Note the two claws (arrow), a distinctive marker of the distal end of the leg. (C) Antp-like. The transformation into leg shown by this clone affects the arista (though part remains untransformed, arrow), and the three (III, II and I) antennal segments as well as structures proximal to the antenna. It closely resembles a dominant Antp transformation (Schneuwly et al., 1987). Most bristles in the clone contains bracts, an epidermal element associated with bristles of the distal leg segments. (D) $l a b$-like. Clone (arrow) in the dorsal posterior head differentiating mesonotal structures, showing a phenotype like that described for $l a b^{-}$ clones (Merrill et al., 1989). (E) Clone in the first abdominal tergite (A1) exhibiting features of a more posterior segment and resembling the A5 pattern. As often occurs with abdominal clones (García-Bellido and Merriam, 1971), some clone bristles have moved away from the clone trichomes (encircled by asterisks). Note that the clone bristles (arrowheads) are bigger than those of A1, the cuticle is more pigmented, and the trichomes are more spaced; compare with surrounding wild-type tissue. (F) Mutant clone (encircled by asterisks) including five bristles (arrowheads) in the dorsal A2 segment showing an A5-like transformation. The clone cuticle is slightly more pigmented and the trichomes more spaced than in surrounding wild-type territory. These features are also seen in the clone in E, indicating that the two clones exhibit the same pattern. This pattern is the same in all the clones in the A1-A5 abdominal segments.

of the A5 tergite (Fig. 1E,F). This suggests that the morphological diversity in A1-A5 depends on exd function and also points to an interaction of exd with Ubx and abd-A, the prime patterning gene products in this area. It is worth noting that 

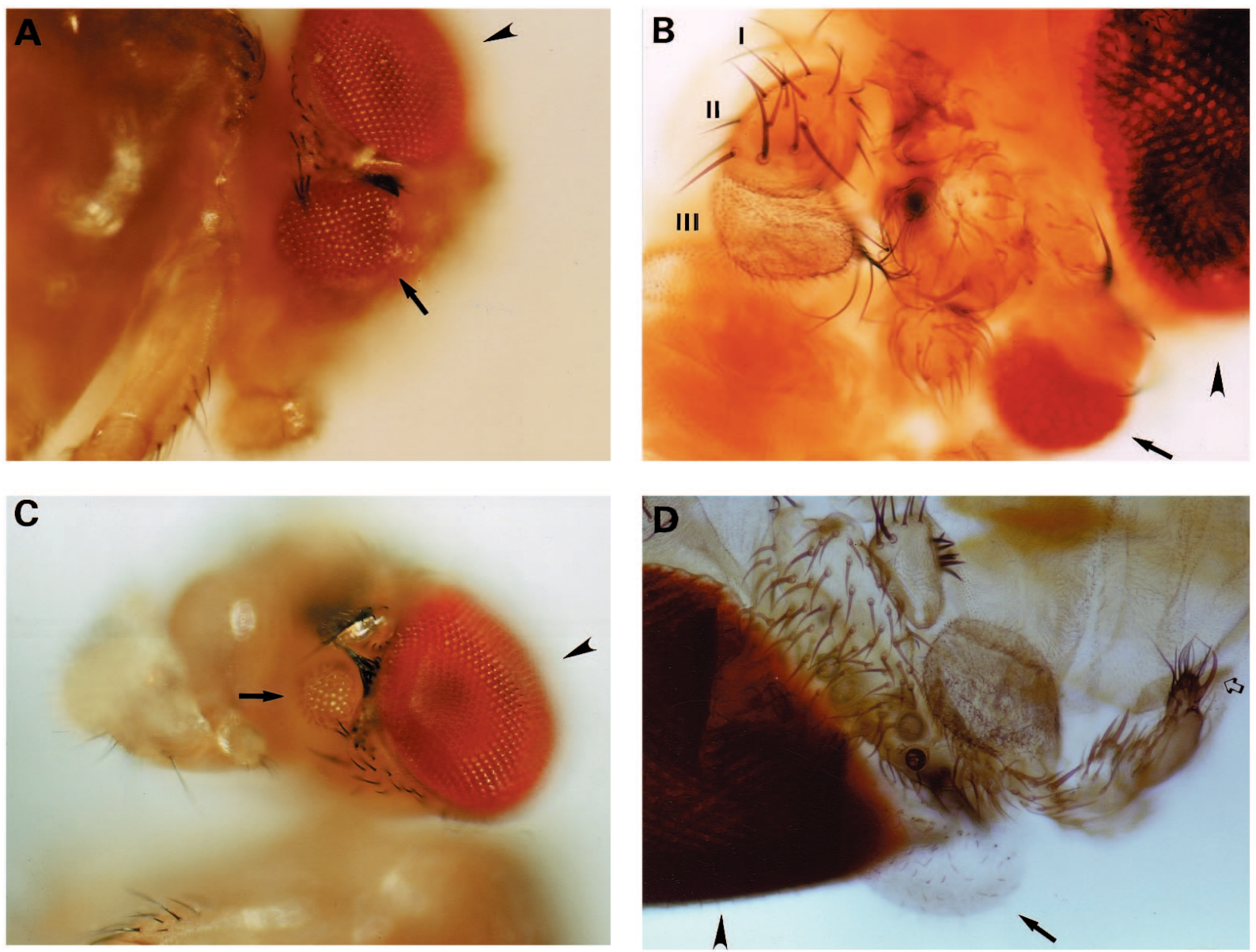

Fig. 2. Ectopic eye formation by $e x d^{-}$clones. (A) Large $y f^{36 a}$ clone differentiating an ectopic eye (arrow) ventral to the normal one (arrowhead). The clones differentiating eye always appear in this region, which normally differentiates the rostral membrane (see Fig. 5). Other structures differentiated by this clone are out of focus. (B) $y f^{36 a}$ clone transforming the ptilinum, a membranous region between the antenna and the dorsal head, into proximal leg (center of the photograph) and also differentiating an ectopic eye (arrow). Note that the clone does not extend to the antenna (antennal segments I, II and III are unaffected), demonstrating that, in addition to the antenna, other head regions have also the potential to produce a leg transformation. Part of the normal eye is visible (arrowhead). (C) $y w f^{36 a}$ clone differentiating an ectopic eye marked with white (arrow), which can easily be distinguished from the normal red eye (arrowhead). (D) A large $y w f^{36 a}$ clone differentiating an ectopic white eye (arrow) and leg $\left(y\right.$ and $\left.f^{36 a}\right)$ containing proximal and distal (note the claw, open arrow) structures. The clone differentiates dorsal segment derivatives like eye, and ventral elements like leg, which appear in inverted orientation with respect to the normal situation. The normal eye (arrowhead and out of focus) is not affected. The existence of clones like this suggests that in the normal eye-antenna disc there are two groups of potential eye primordia in opposite orientation.

clones in the A1 segment, the exclusive domain of $U b x$, develop the same pattern as those in A2-A4 where $a b d-A$ is expressed and has a predominant function, suggesting that in the absence of $e x d, U b x$ and $a b d-A$ have equivalent roles (see Discussion).

Another example of novel homeotic transformation is the phenotype of $e x d^{-}$clones in ventral head regions ( $\left.n=57\right)$, many of which also extend to the antenna. Clones appearing in the rostral membrane differentiate ectopic eyes (Fig. 2A,C) and, if they extend dorsally to the ptilinum (a membrane between the anterior dorsal head and the antenna, see Fig. 5E), in addition, they differentiate proximal and medial leg structures, which can be identified as of midleg identity (Fig. 2B). This is different from the aristapedia or Antennapedia-like transformations mentioned above as we often observe this transformation in clones that do not reach the antenna (Fig. 2B). As there is no lineage restriction in the eye antenna disc until nearly the third larval period (Morata and Lawrence, 1979), early clones may transform the antenna, the ptilinum and the rostral membrane producing proximal, medial and distal leg in addition to an ectopic eye (Fig. 2D).

\section{exd- clones eliminate the dorsoventral difference in the abdomen}

The lack of exd function transforms the ventral abdomen into dorsal abdomen. Mutant clones $(n=48)$ that appear in the sternites (the ventral abdominal structures) differentiate as tergites (dorsal abdominal structures) (Fig. 3A). That is, in the absence of $\operatorname{exd}$ function, ventral abdominal cells acquire dorsal identity, e.g. the adult equivalent of the dorsal phenotype (Nusslein-Volhard, 1979). Since the dorsoventral segregation occurs earlier than individual segment specification, this result suggests that $e x d$ is involved with the maintenance of a developmental function that precedes segment determination. As 

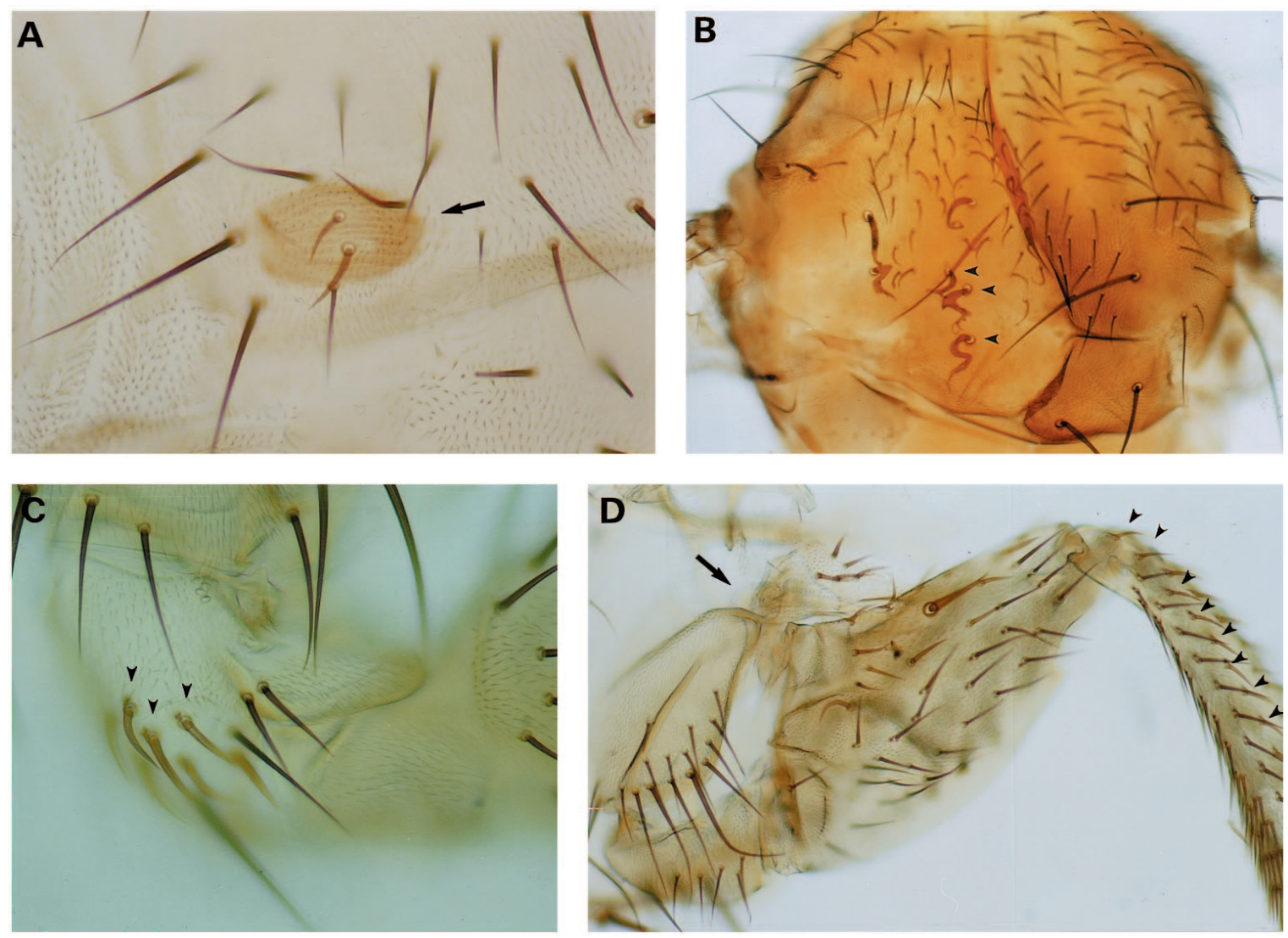

Fig. 3. Non-homeotic transformations in $\operatorname{exd}^{-}$clones. (A) Mutant clone (arrow) in the ventral abdomen (sternite) of A5 showing a transformation to dorsal (tergite) as indicated by the pigmentation of cuticle, the type of trichomes and the spacing and type of bristles. All the clones found in the sternites in the A1-A5 region show a similar transformation. In the A6 sternite, the clones also show a transformation into tergite but, in this case, they differentiate 1-3 bristles surrounded by very few or no trichomes. This is just what is expected for a transformation into the sixth tergite, which is mostly devoid of trichomes. (B) Large exd ${ }^{-}$clone in the mesonotum showing an abnormal disposition of macrobristles (arrowheads) as compared with the normal pattern to the right. Note the absence of scutellar bristles, the apparent duplication of dorsocentrals and the lack of infralar bristles. This is the stereotyped pattern of the $e x d^{-}$clones in this region. (C) Small clone in a proximal leg segment (trochanter) differentiating bristles characteristic of a more distal leg segment, probably tibia. Note the presence of bracts (arrowheads) associated with the $y f^{36 a}$ bristles whereas the nearby wild-type bristles do not possess them. In wild-type legs, bracts are present in part of the femur, tibia, basitarsus and tarsal segments, but are always absent in proximal femur, trochanter, coxa and pleura. exd- clones in the trochanter, proximal femur and, sometimes in the coxa, differentiate bracts and bristle types characteristic of more distal leg segments, indicating a change in identity along the proximodistal axis. (D) A typical large clone in the proximal leg. It extends from the pleural membrane to the distal femur and tibia where it differentiates according to the normal pattern, as indicated by the clone bristles (arrowheads) in the tibia. But notice the physical continuity of the clone through a bridge of material (arrow) to the proximal femur, by-passing the coxa and trochanter. The leg is joined to the pleura in two places, the coxa and the proximal femur and, as a result, all leg structures in between are stuck to the pleura.

embryos lacking both maternal and zygotic exd product do not show a parallel transformation (Peifer and Wieschaus, 1990), the requirement for exd appears to be restricted to imaginal cells. Within the imaginal cells, it is further restricted to the abdomen, for $e x d^{-}$clones in the thoracic segments fail to show a ventral-to-dorsal transformation.

\section{exd has a role in the generation of subpatterns within several imaginal discs}

There are two cases in which the $\operatorname{exd}^{-}$clones produce alterations not associated with segmental transformations, but restricted to discrete regions within a single imaginal disc. In the wing disc derivatives, exd $^{-}$clones $(n=90)$ differentiate in the mesonotum bristles characteristic of this region, but these are arranged in an abnormal pattern (Fig. 3B) and some characteristic macrobristles are missing. This may suggest an involvement of exd in the positioning and/or bristle determination in the thorax. In most of the wing blade, the clones proliferate and differentiate normally except those in the hinge region, which produce unusual bristled patterns and outgrowths unlike any normal pattern (not shown).

Clones in the three legs also show an effect that depends on the position where they appear. Clones in the proximal regions (pleura, coxa, trochanter and proximal femur) $(n=99)$ differentiate inappropriate patterns, which, especially in the trochanter and femur, can be shown to correspond to more distal leg segments. In contrast, clones in the distal half of the femur, tibia, basitarsus and tarsus differentiate normally (Fig. 3C,D). Thus, exd function is only required in the proximal leg segments.

\section{exd expression in imaginal discs}

All the experiments described above indicate that exd is 
expressed in the precursors (imaginal discs) of many adult segments. However, the effect of exd clones is often restricted to part of the derivatives of the corresponding disc, indicating a localized requirement and in turn suggesting the possibility of spatially restricted exd expression. Using a specific anti-exd antiserum produced in our laboratory (see Materials and Methods), we have immunolocalized the exd protein in the different imaginal discs.

We find that, in contrast to the reported distribution of exd transcripts (Flegel et al., 1993), the exd protein exhibits a localized distribution in the imaginal discs (Fig. 4). In the eyeantenna disc, exd is present in most part of the antennal region spanning the first, second and third antennal segments (Fig. $4 \mathrm{~A})$, but part of the third antennal segment and the arista region are devoid of product; in the eye part, exd localizes to the region that surrounds anteriorly the eye precursors, including the postgena, the ptilinum and precursors of the head capsule (Fig. 4A) (Haynie and Bryant, 1986). In the wing disc, the exd protein is present in the notum region and in the area corresponding to the wing hinge and is absent in the wing pouch (Fig. 4B) (Bryant, 1975). A similar pattern is found in the haltere disc (data not shown). In the leg discs, the expression is restricted to the periphery, where the precursors of proximal leg segments such as coxa, trochanter and femur are located, whereas the central disc region, differentiating tibia, basitarsus and tarsal segments appear devoid of exd protein (Fig. 4C,D) (Fristrom and Fristrom, 1993).

\section{DISCUSSION}

\section{Distinct roles of exd in adult patterning}

Using genetic mosaics, we have constructed a piecemeal description of the adult exd phenotype, that is, of the adult patterns as specified in the absence of the exd gene product. As summarised in Fig. 5, they differ greatly from the normal patterns, illustrating the extent of exd contribution.

The first conclusion from our results is that exd is involved in specifying the identities of adult segments, in parallel with what is observed for the embryonic segments (Peifer and Wieschaus, 1990). Mutant exd $d^{-}$clones produce homeotic transformations resembling those produced by homeotic mutations
A
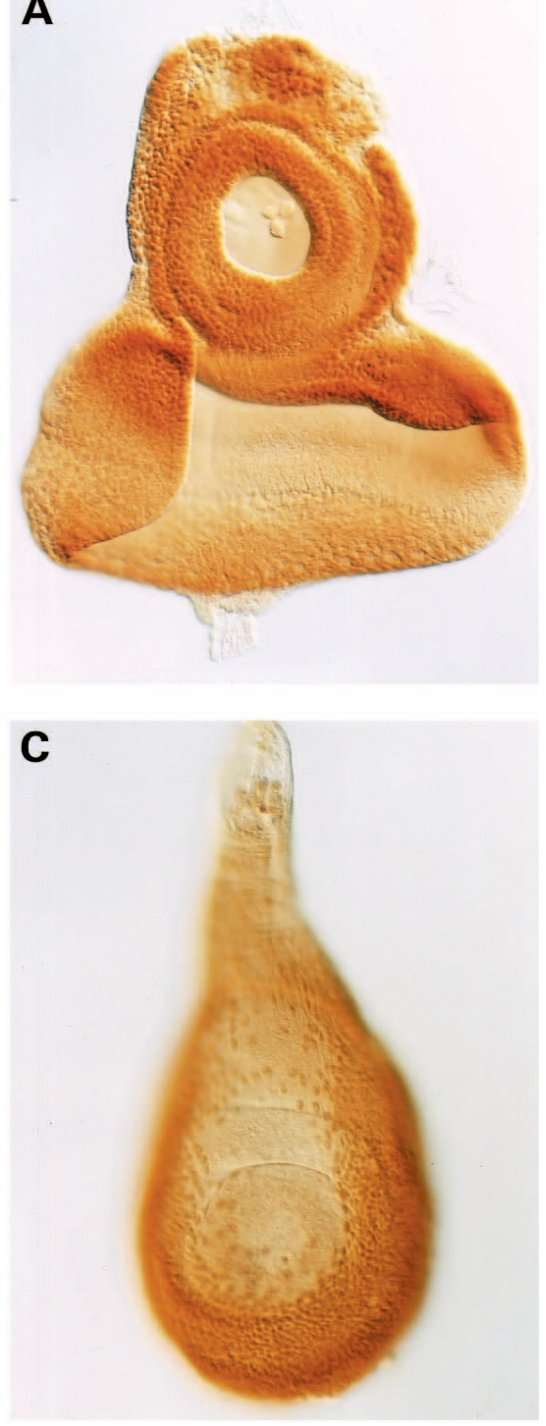

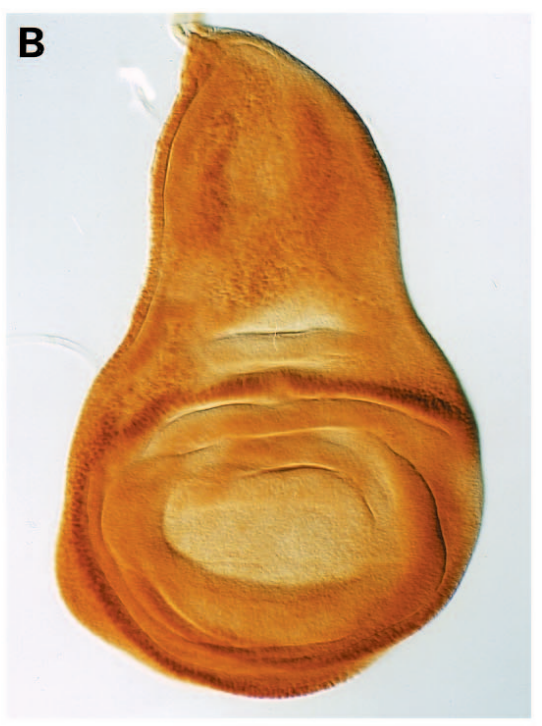

D

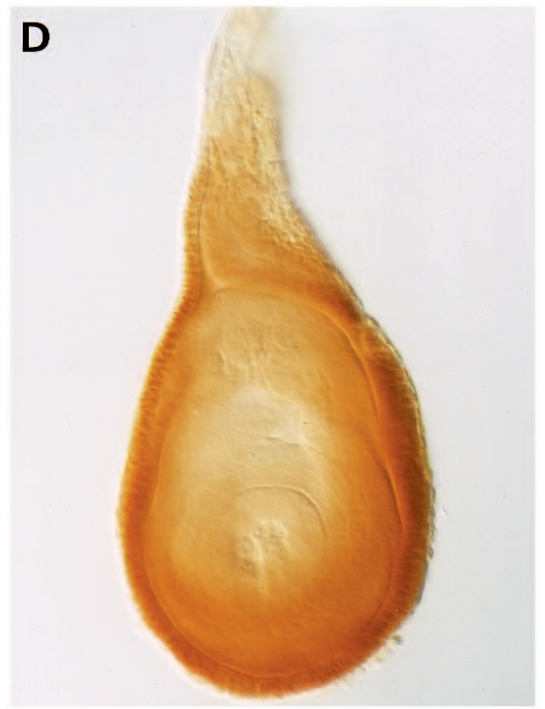

Fig. 4. Immunolocalization of exd in imaginal dics. (A) Eye-antenna disc. Nuclear staining is restricted to the antenna region and the surroundings of the eye primordium. No staining is detected in the eye region. In the antenna, exd protein is localized in the first, second and part of the third antennal segments, but no apparent staining is observed in the arista (out of focus). The outer region also shows staining and contains the precursors of the posterior head. In the region surrounding the eye primordium, exd protein is localized in the region precursor of the postgena, the ptilinum and the orbital and frontal regions (Haynie and Bryant, 1986). (B) Wing disc. Strong nuclear staining appears in a circle around the wing pouch corresponding to the hinge region. Additional weaker staining is detected in two patches located in the notum region. There is no detectable staining in the wing pouch (Bryant, 1975). (C,D) Leg disc. Two different focal planes of the same disc are shown. In $\mathrm{C}$, the apical surface is in focus. exd protein is detected in the outer part of the disc, which is precursor of the proximal segments of the leg including coxa, trochanter and femur. Staining is reduced in the central region, which corresponds to the peripodial epithelium, although some nuclei show staining. In D, the basal surface is in focus. Again, staining is detected in the outer ring of the disc, precursor of the proximal leg. No staining is observed in the central regions, which are precursors of distal segments such as tibia and tarsi (Fristrom and Fristrom, 1993). 
like Ubx, labial or Antennapedia. Even the transformations in the A1-A5 abdominal segments, which do not resemble any known homeotic mutation, affect the identity of the segments along the anteroposterior body axis. Although the transformations observed in the head are more difficult to interpret in terms of segmental determination due to its complex segmental organization, the fact that some clones produce dorsal (ectopic eyes) and ventral (leg) segmental structures also suggests a segmental transformation along the anteroposterior axis. It also supports the view (Jürgens et al., 1986) that the derivatives of
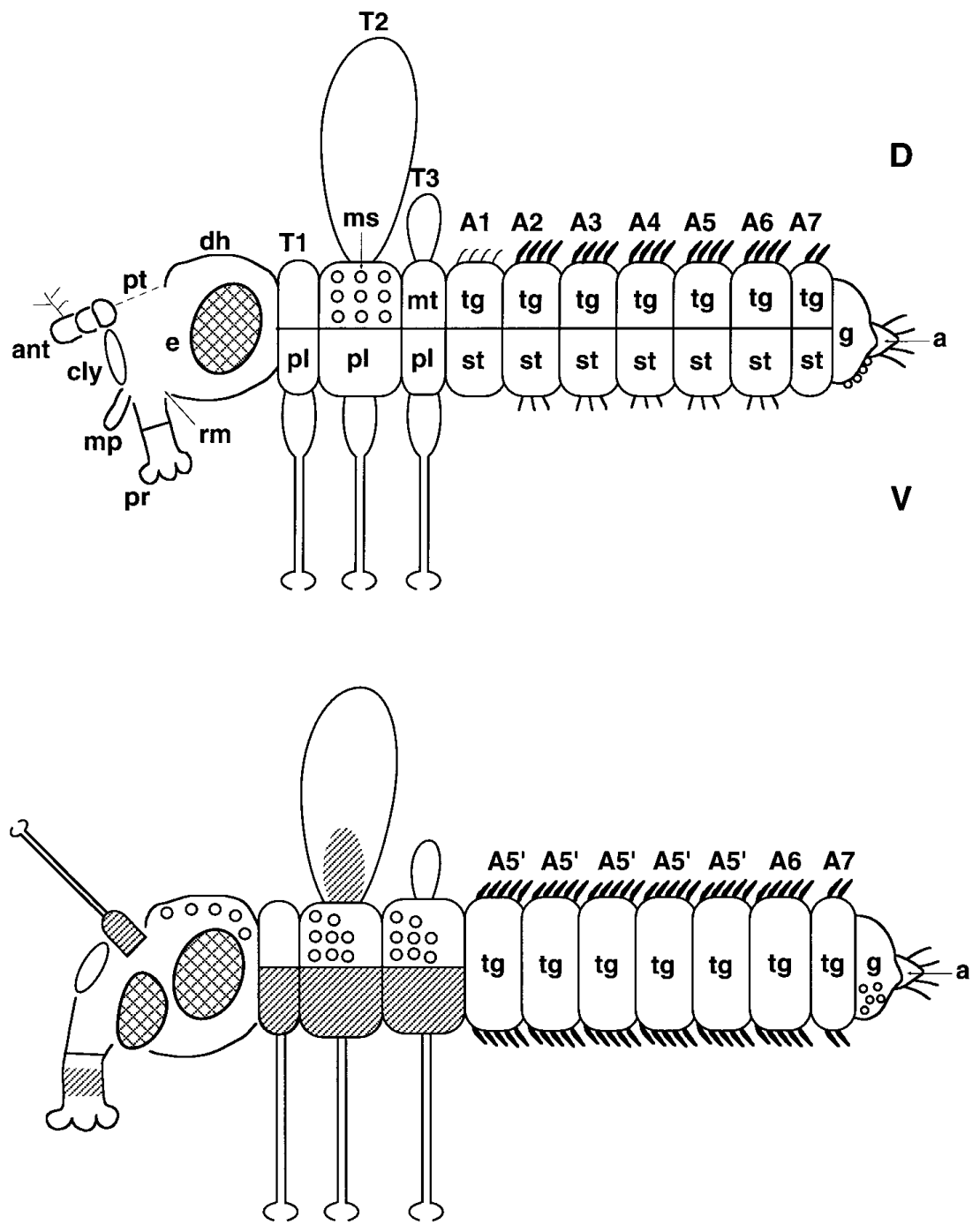

Fig. 5. Wild-type versus $e x d^{-}$adult Drosophila. Scheme of a wild type (top) and an $e x d^{-}$(bottom) as reconstructed by the phenotype of $e x d^{-}$clones all over the body of adult females to illustrate the different aspects of exd function. The figure at the bottom portrays the adult patterns as they would be directly specified by the homeotic genes without the modifications introduced by their interactions with exd. The head is formed by the derivatives of three imaginal discs, though the emphasis is put on the structures derived from the eye-antenna disc: dorsal head (dh), eye (e), ptilinum (pt), rostral membrane (rm) antenna (ant) and maxillary palp ( $\mathrm{mp}$ ). The $\mathrm{pt}$ is represented by a dashed line because it is normally folded between the ant and the dh. The two other head discs are the labial disc, which forms the proboscis (pr), and the clypeo-labral disc, which differentiates the clypeus (cly). The three thoracic segments (T1, T2 and T3) and the seven abdominal segments (A1-A7) present in females, in addition to genitalia (g) and analia (a) are shown. The horizontal line spanning the whole trunk of the body represents the boundary between the dorsal (tergite, $\operatorname{tg}$ ) and the ventral (sternite, st) segment derivatives. In the three thoracic segments, the D/V line separates the derivatives of the humeral (T1), wing (T2) and haltere (T3) discs from the corresponding three leg discs. The wing and haltere discs differentiate a proximal component, mesonotum (ms) and metanotum (mt), respectively, as well as the corresponding appendage. The characteristic bristle pattern of the $\mathrm{ms}$ is indicated by the regular array of open circles. In the three legs, we distinguish a trunk component, the pleura, pl, a proximal part of the appendage (coxa, trochanter and proximal part of the femur), represented by a thick sector in the scheme, and a distal region of the appendage (distal femur, tibia, basitarsus and tarsus), represented by the narrow sector. In the abdominal segments, the bristle pattern of tg is very different from that of st. The A1 tg differs from the more posterior ones in bristle size and other aspects. The other tergites can also be distinguished, but their differences are not represented. The principal transformations defined by the $e x d^{-}$clones are illustrated in the bottom figure and are for the most part self-explanatory. Those places where the clones differentiate abnormal patterns that cannot be classified as homeotic transformations are shadowed (proximal wing and leg pleurae). Part of the pr is affected by exd clones which form sac-like structures. In the cly, no clone was detected, probably because they could only be found if transformed into structures in which the markers $y, w$ or $f^{36 a}$ can be detected. In the rest of the head, exd $d^{-}$clones show several distinct transformations. The dh is transformed into ms, and an ectopic leg appears as a result of the transformation of the pt and the ant, whereas an ectopic eye appears in the rm. One implication of this result is that the derivatives of the eye-antenna disc originate from the fusion of several embryonic segments because we can distinguish at least two regions that are in inverted orientation and that appear to represent distinct segment primordia (Jürgens et al., 1986). In the T2 dorsal segment, the effect is restricted to the proximal wing and to the abnormal patterning of bristles in the ms, indicated by different arrangement of open circles. The numerous clones found in the distal wing are entirely normal. In the dorsal T3 segment, the mt becomes transformed into ms supposedly with the same bristle arrangement, but the haltere appendage is not transformed. The three legs are equally modified; the distal portions (from the tibia towards distal) are entirely normal, but more proximal segments are altered. The femur and trochanter develop distal tibia-like patterns and the transformations of pleura and coxa cannot be assigned. In the abdomen, the st are transformed into tg eliminating the dorsoventral distinction. The tg corresponding to A1-A5 segments develop a pattern resembling, though not exactly identical to, that of A5. The A6 and A7 tg identities are not detectably altered. The effect of the clones in the genitalia ( $\mathrm{g}$ ) has not been studied in detail, but bristle patterning is abnormal. The analia (a) is not altered. 
the eye-antenna disc result from the fusion of at least two embryonic segments, which we find are in inverted orientation (see legend to Fig. 5). As segment determination along the anteroposterior axis is a principal function of the primary homeotic genes (Sánchez-Herrero et al., 1985; Kaufman et al., 1990), all these observations suggest the existence of interactions between exd and those genes (see below).

Nevertheless, it is also clear from our work that exd participates in other developmental events that are not directly connected with the determination of segment patterns along the anteroposterior body axis. Most notable is the finding that $\mathrm{exd}^{-}$ clones affect dorsoventral patterning in the abdomen, pointing to an early developmental restriction that is retained by adult cells and which requires exd function. This indicates a hitherto unsuspected mechanism to maintain the embryonic dorsoventral polarity in adult cells.

The function of exd is also necessary for other developmental events that affect discrete regions of adult segments and which occur in late developmental stages. In the legs, exdclones do not change the segmental identity, but transform proximal leg regions into more distal ones, thus indicating an involvement of exd in proximodistal polarity of the appendage. Similarly, exd is necessary in certain regions of the T2 segment but not in others; in the distal wing regions, exd $^{-}$clones have no effect, whereas they produce pattern alterations in the mesonotum and wing hinge.

The picture that emerges from all these observations is that, regarding adult patterns, exd is involved in several distinct developmental events: the correct determination of several cephalic, thoracic and abdominal segments, the maintenance of the embryonic dorsoventral polarity, the specification of normal identity along the proximodistal axis of the legs and, perhaps, the positioning of bristles in the thorax.

\section{The adult expression of exd}

The exd product is present in all the imaginal discs that we have examined with the specific anti-exd antibody. We have not looked at the abdominal histoblasts, but the behavior of the mutant clones demonstrates that exd product must be present at least in some portions of the abdominal segments. However, even though the protein is present in all imaginal discs, the product distribution within them is not generalized, but restricted to certain regions that coincide with the areas where the $e x d^{-}$clones have a phenotypic effect. The clearest examples are the wing and the leg discs, where the effect of the mutant clones in the proximal regions (or the lack of effect in the distal ones) matches with the presence (or absence) of product in the corresponding zone of the disc according to the fate map.

In the eye-antenna disc, however, the situation is more complicated. We find the exd product is lacking in the most distal region, corresponding to part of the third antennal segment and the arista, even though we recover in this region mutant clones that produce leg transformations. The reasons for this are not clear, but it is possible that only a fraction of the antenna cells are transformed by the mutant clones. We are presently studying the distribution of all the antennal clones to answer this question.

\section{The mode of action of exd}

The general view about the mode of action of exd is that it acts as a cofactor of homeotic function, as suggested by Peifer and Wieschaus (1990). Some of the phenotypes observed in our clones strongly support this view: the transformation of metanotum into mesonotum, identical to that produced by the lack of $U b x$ function, suggests that $U b x$ function is mediated by exd and is fully consistent with recent work showing that the exd product enhances the affinity of Ubx protein for specific DNA binding (Chan et al., 1994; van Dijk and Murre, 1994). Likewise, the lab-like phenotype of $e x d^{-}$clones in the dorsal head suggests that the lab product requires the exd product for efficient DNA binding. Similarly, eye transformations found in the rostral region may indicate an interaction with the homeobox genes eyeless (ey) (Quiring et al., 1994) and/or sine oculis (so) (Cheyette et al., 1994).

The $e x d^{-}$phenotype in the A1-A5 tergites is also consistent with the cofactor hypothesis, for it suggests an interaction of exd with $U b x$ and with $a b d-A$. One interesting aspect of this transformation is that the $e x d^{-}$pattern is the same in all these segments, even though they differ with respect to $U b x$ and $a b d$ $A$ expressions. One possibility is that in the absence of exd function, abdominal genes like $U b x$ and $a b d-A$ determine the same "ground" abdominal pattern, which is normally refined through their specific interactions with $e x d$. The implication of this hypothesis is that the functions of $U b x$ (responsible for the A1 pattern) and of $a b d-A$ (primarily responsible for A2-A4 patterns) are equivalent; it is their distinct interaction with exd that makes the difference (Lawrence and Morata, 1994). Finally, the effect of exd clones in the proximal leg structures may imply an interaction of exd with Distal-less (Dll), another homeobox gene necessary for the proximodistal patterning in appendages (Cohen et al., 1989).

However, it is possible that the exd product acts not only as cofactor of homeoproteins but through other mechanisms as well. In our interpretation we have assumed, from the work performed in embryos (Peifer and Wieschaus, 1990), that the elimination of exd does not alter the normal expression patterns of homeotic genes, but this has not been demonstrated for imaginal cells. Some of the effects that we see in exd $^{-}$clones could be due to a loss of the transcriptional control of homeotic genes by exd. For example, the development of ectopic eyes observed in our experiments probably requires $e y^{+}$function (Quiring et al., 1994). This may result from inappropriate activation of $e y^{+}$in the rostral region, or alternatively, the ey product may be normally present there but functionally suppressed by the exd product. A similar argument can be made for the dominant Antp-like phenotype of the clones in the head; it may indicate a gain-of-function of the Antp gene, which is not normally expressed in the eye-antenna disc (Wirz et al., 1986), in turn suggesting transcriptional control. It is also possible that the exd product may interact with transcription factors other than homeoproducts. For example, no homeotic function has so far been related with the embryonic dorsoventral restriction and yet exd function is necessary for its maintenance. Similarly, no homeotic function has been proposed to act in the arrangement of bristles in the thorax, another process in which exd appears to play a role. Finally, since exd itself encodes a homeoproduct, it may have a regulatory role of its own, not requiring other factors.

Given the extraordinary evolutionary conservation of exd (the homology with the human $p b x 1, p b x 2$ and $p b x 3$ genes (Nourse et al., 1990; Kamps et al., 1990; Monica et al., 1991) 
is about $71 \%$ in the whole sequence and nearly $100 \%$ in the homeodomain (Flegel et al., 1993; Rauskolb et al., 1993)), it is possible that the diverse morphogenetic function of exd observed in Drosophila may be present in other animals. It would be of great interest to study the function of the vertebrate homologs of exd. We would expect that some Hox gene functions may be mediated by interactions with $p b x$ genes in mammals.

We thank Cordelia Rauskolb and Eric Wieschaus for fly stocks and plasmids, Juan Botas for fly stocks, Rosa González for expert technical assistance, Ernesto Sánchez-Herrero, Isabel Guerrero and Peter Lawrence for critically reading the manuscript and all the members of the Morata's laboratory for help and encouragement. S. G-C. is a postdoctoral fellow from the Spanish Ministerio de Educación y Ciencia. This work was supported by grants from the Dirección General de Investigación Científica y Técnica and the Human Frontier Science Program Organization and an institutional grant from the Fundación Ramón Areces. S. G-C. dedicates this paper to Michael Levine on his 40th birthday.

\section{REFERENCES}

Bryant, P. J. (1975). Pattern formation in the imaginal wing disc of Drosophila melanogaster: Fate map, regeneration and duplication. J. Exp. Zool. 193, 4978.

Casanova, J., Sánchez-Herrero, E. and Morata, G. (1987). Double and triple mutant combinations for the bithorax complex of Drosophila. EMBO J. 6, 3103-3109

Chan, S., Jaffe, L., Capovilla, M., Botas, J. and Mann, R. M. (1994). The DNA binding specificity of Ultrabithorax is modulated by cooperative interactions with Extradenticle, another homeoprotein. Cell 78, 603-615.

Cheyette, B. N. R., Green, P. J., Martin, K., Garren, H., Hartenstein, V. and Zipursky, S. L. (1994). The Drosophila sine oculis locus encodes a homeodomain-containing protein required for the development of the entire visual system. Neuron 12,977-996.

Cohen, S. M. (1993). Imaginal disc development. In The Development of Drosophila melanogaster (eds. M. Bate and A. Martínez-Arias), pp. $747-$ 841. New York: Cold Spring Harbor Laboratory Press.

Cohen, S. M., Brönner, G., Küttner, F., Jürgens, G. and Jäckle, H. (1989). Distal-less encodes a homoeodomain protein required for limb development in Drosophila. Nature 338, 432-434.

Desplan, C., Theis, J. and O'Farrell, P. H. (1985). The Drosophila developmental gene engrailed encodes a sequence specific DNA binding activity. Nature 318, 630-635.

Desplan, C., Theis, J. and O'Farrell, P. H. (1988). The sequence specificity of homeodomain-DNA interaction. Cell 54, 1081-1090.

Flegel, W. A., Singson, A. W., Margolis, J. S., Bang, A. G., Posakony, J. W. and Murre, C. (1993). Dpbx, a new homeobox gene closely related to the human proto-oncogene pbxl. Molecular structure and developmental expression. Mech. Dev. 41, 155-161.

Fristrom, D. and Fristrom, J. W. (1993). The metamorphic development of the adult epidermis. In The Development of Drosophila melanogaster (eds. M. Bate and A. Martínez-Arias), pp. 843-897. New York: Cold Spring Harbor Laboratory Press.

García-Bellido, A. and Merriam, J. R. (1971). Clonal parameters of tergite development in Drosophila. Dev. Biol. 26, 264-276.

González-Reyes, A. and Morata, G. (1990). The developmental effect of overexpressing a $U b x$ product in Drosophila embryos is dependent on its interactions with other homeotic products. Cell 61, 515-522.

Guan, K. and Dixon, J. E. (1991). Eukaryotic proteins expressed in Escherichia coli: An improved thrombin cleavage and purification procedure of fusion proteins with glutathione $S$-transferase. Anal. Biochem. 192, 262267.

Haynie, J. L. and Bryant, P. J. (1986). Development of the eye-antenna imaginal disc and morphogenesis of the adult head in Drosophila melanogaster. J. Exp. Zool. 237, 293-308.

Hoey, T. and Levine, M. (1988). Divergent homeo box proteins recognize similar DNA sequences in Drosophila. Nature 332, 858-861.
Jürgens, G., Lehman, R. Schardin, M. and Nüsslein-Volhard, C. (1986). Segmental organisation of the head in the embryo of Drosophila melanogaster. A blastoderm fate map of the cuticle structures of the larval head. Roux' s Arch. of Dev. Biol. 195, 359-377.

Kamps, M. P., Murre, C., Sun, X. and Baltimore, D. (1990). A new homeobox gene contributes the DNA binding domain of the $t(1 ; 19)$ translocation protein in pre-B ALL. Cell 60, 547-555.

Kaufman, T. C., Seeger, M. A. and Olsen, G. (1990). Molecular and genetic organization of the Antennapedia gene complex of Drosophila melanogaster. Adv. Genetics 27, 309-362.

Kerridge, S. and Morata, G. (1982). Developmental effects of some newly induced Ultrabithorax alleles of Drosophila. J. Embryol. Exp. Morph. 68, 211-234.

Lawrence, P. A. and Morata, G. (1994). Homeobox genes: Their function in Drosophila segmentation and pattern formation. Cell 78, 181-189.

Lewis, E. B. (1978). A gene complex controlling segmentation in Drosophila. Nature 276, 565-570.

Merrill, V. K. L., Diederich, R. J., Turner, F. R. and Kaufman, T. C. (1989) A genetic and developmental analysis of mutations in labial, a gene necessary for proper head formation in Drosophila melanogaster. Dev. Biol. 135, 376-391.

Monica, K., Galili, N., Nourse, J., Saltman, D. and Cleary, M. L. (1991) $P B X 2$ and $P B X 3$, new homeobox genes with extensive homology to the human proto-oncogene PBX1. Mol. Cell. Biol. 11, 6149-6157.

Morata, G. and Lawrence, P. A. (1977). Homoeotic genes, compartments and cell determination in Drosophila. Nature 265, 211-216.

Morata, G. and Lawrence, P. A. (1979). Development of the eye-antenna imaginal disc of Drosophila. Dev. Biol. 70, 355-371.

Morata, G. and Ripoll, P. (1975). Minutes: Mutants of Drosophila autonomously affecting cell division rata. Dev. Biol. 42, 211-221.

Nourse, J., Mellentin, J. D., Galili, N., Wilkinson, J., Stanbridge, E., Smith, S. D. and Cleary, M. L. (1990). Chromosomal translocation $t(1 ; 19)$ results in synthesis of a homeobox fusion mRNA that codes for a potential chimeric transcription factor. Cell 60, 535-545.

Nüsslein-Volhard, C. (1979). Maternal effect mutations that alter the spatial coordinates of the embryo of Drosophila melanogaster. In Determinants of Spatial Organization, (ed. I. Koenigsberg and S. Subtelney), pp. 185-211. New York: Academic Press.

Peifer, M. and Wieschaus, E. (1990). Mutations in the Drosophila gene extradenticle affect the way specific homeo domain proteins regulate segmental identity. Genes Dev. 4, 1209-1223.

Postlethwait, J. H. and Girton, J. R. (1974). Development in genetic mosaics of aristapedia, a homoeotic mutant of Drosophila melanogaster. Genetics 76, 767-774.

Quiring, R., Walldorf, U., Kloter, U. and Gehring, W. J. (1994). Homology of the eyeless gene of Drosophila to the Small eye gene in mice and Aniridia in humans. Science 265, 785-789.

Rauskolb, C., Peifer, M. and Wieschaus, E. (1993). extradenticle, a regulator of homeotic gene activity, is a homolog of the homeobox-containing human proto-oncogene pbx1. Cell 74, 1101-1112.

Sánchez-Herrero, E., Guerrero, I., Sampedro, J. and González-Reyes, A. (1994). Developmental consequences of unrestricted expression of the $a b d-A$ gene of Drosophila. Mech. Dev. 46, 153-167.

Sánchez-Herrero, E., Vernós, I., Marco, R. and Morata, G. (1985) Genetic organization of Drosophila bithorax complex. Nature 313, 108-113.

Schneuwly, S., Klemenz, R. and Gehring, W. J. (1987). Redesigning the body plan of Drosophila by ectopic expression of the homoeotic gene Antennapedia. Nature 325, 816-818.

Smith, D. B. and Johnson, K. S. (1988). Single-step purification of polypeptides expressed in $E$. coli as fusions with glutathione S-transferase. Gene 67, 31-40.

Thali, M., Müller, M. M., DeLorenzi, M., Matthias, P. and Bienz, M. (1988). Drosophila homoeotic genes encode transcriptional activators similar to mammalian OTF-2. Nature 336, 598-601.

van Dijk, M. A. and Murre, C. (1994). extradenticle raises the DNA binding specificity of homeotic selector gene products. Cell 78, 617-624.

Wilson, D. S. and Desplan, C. (1995). Cooperating to be different. Curr. Biol. 5, 32-34.

Wirz, J., Fessler, L. I. and Gehring, W. J. (1986). Localization of the Antennapedia protein in Drosophila embryos and imaginal discs. EMBO J. 5, 3327-3334 